\title{
Usability of car stereo
}

\author{
Razza, Bruno Montanari ${ }^{\mathrm{a},}$, Paschoarelli, Luis Carlos ${ }^{\mathrm{b}}$ \\ ${ }^{a}$ Department of Design and Fashion, Maringa State University, R. Dom Pedro II, s/n, Cianorte (PR), Brazil. \\ ${ }^{\mathrm{b}}$ PPGDeisng, FAAC, São Paulo State University - Av. Eng. Luis E. C. Coube, 14-01, 17033-360, Bauru, Brazil
}

\begin{abstract}
Automotive sound systems vary widely in terms of functions and way of use between different brands and models what can bring difficulties and lack of consistency to the user. This study aimed to analyze the usability of car stereo commonly found in the market. Four products were analyzed by task analysis and after use reports and the results indicate serious usability issues with respect to the form of operation, organization, clarity and quality of information, visibility and readability, among others.
\end{abstract}

Keywords: Keyword one, keyword two, keyword three, keyword four, keyword five

\section{Introduction}

Usability is a recent field of ergonomics, having its beginnings around the late 1980s [1, 4]. The ISO 9241-11 [2] defines it as the extent to which a product can be used by specified users to achieve specified goals with effectiveness, efficiency and satisfaction in a specified context of use.

In general, car stereos have an interface that is not intuitive to novice users and the lack of consistency is evident since there is a great variation in their layout and functions among brands. Jordan [3] presented pleasurable e displeasurable experiences with products and the user of a car stereo reported that she felt so frustrated with the product that she finally became resigned to her car stereo being difficult to use. This situation is not restricted to this report but instead seems to be a recurring problem.

The interface of the car stereo needs to be particularly easy to use due to its context of use. Since the user of the stereo are also drivers, the time consumed to scan for function and accomplish the task must be minimal in order not to divert user's attention from the primary task (driving). Zwalen et al. [5] have made a study to measure the amount of eye fixations made to use a car stereo while driving and consequently the eye deviations from the road. The results showed that many tasks operated in the radio are considered an inacceptable risk for the user, since they demand a great number of eye deviations and the fixations are too long. Some of those tasks are, for example, scan for stations manually or automatically.

The objective of this study was to investigate the usability of some sound devices commonly found in Brazilian market and focusing on user's performance and perception.

\section{Method}

Four car stereo models (all radio and MP3 CD players) were selected for usability evaluation (Fig. 1). Twenty eight volunteers of both genders (18 - 35 years old) took part in the usability assessment, with seven different individuals for each product. Exclusion criteria consisted of users who reported great intimacy with some specific model (in order to avoid the influence of previous experience); individuals with visual deficiencies who were not using corrective lenses; and people with cognitive or neuro-motor weaknesses. Written consent was taken for all subjects.

Previously to all analysis a questionnaire was applied to identify the user's level of experience in car stereos. Then the subject was asked to sit in the driver's seat of the vehicle to perform the tasks. The car was parked, ideal lighting conditions was provided and the presence of observers was avoided. An MP3 $\mathrm{CD}$ with songs organized into folders was employed

\footnotetext{
*Corresponding Author. E-mail: bmrazza@uem.br.
} 
and the users' performance was recorded in a digital camera.

The user was asked to perform four different tasks, as follows:

- Task 1: set to play the third song of the second CD MP3 folder - the radio was turned off and without the panel. If necessary, the volume could be changed.

- Task 2: switch to radio and tune in a station that is not recorded in product memory;

- Task 3: back to the CD, play the fifth song of the first folder and put the display to show the name of the song;

- Task 4: change the type of sound (low/high/ presets) and other advanced display which may vary according to the model analyzed.

No instruction of how to perform the task was given unless the subject clearly stated that he/she did not know how to perform the task. The subject was also encouraged to give comments about the task performed and the product. The audiovisual recordings were later analyzed in order to identify the incorrect actions taken and the pauses between operations (for pauses were consider a significant time taken between operations and errors were stated for each click on the wrong button).

After the performed tasks, subjects were asked about their perception regarding the product usability. An analog scale was used to rate the following criteria: satisfaction; ease of use; information comprehensibility; buttons layout; visual quality of the display; and aesthetics.

\section{Results and discussion}

There were more errors and more breaks in the first task, due to unfamiliarity with the product in use
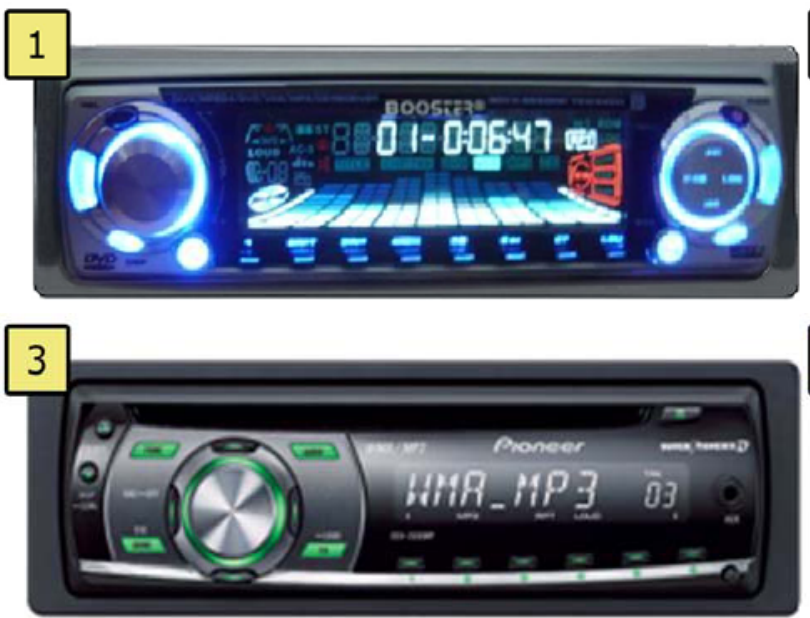

(Table 1). Thus, we can state that the knowledge gained in the first task allowed the others to be more easily accomplished.

Table 1.

Task analysis results

\begin{tabular}{llcccc}
\hline \multirow{2}{*}{ Task 1 } & & A & B & C & D \\
\cline { 2 - 6 } & Pauses & 15 & 6 & 9 & 11 \\
\cline { 2 - 6 } Task 2 & Errors & 19 & 10 & 16 & 23 \\
\hline & Pauses & 1 & 9 & - & 11 \\
\cline { 2 - 6 } Task 3 & Errors & 4 & 9 & 6 & 18 \\
\hline Task 4 & Pauses & 10 & 9 & 6 & 12 \\
\cline { 2 - 6 } & Errors & 2 & 9 & 3 & 15 \\
\cline { 2 - 6 } & Eauses & 3 & 7 & 6 & 7 \\
\hline
\end{tabular}

A - Booster BDVX-5580MP

B - Pioneer DEH-2000MP

C - Pioneer Mosfet50

D - Sony CTX-GT210

The entire sample received negative evaluations for most of the criteria, and especially for the problems related to information comprehensibility, display quality, ease of use and buttons layout (Table 2). Analysis of the answers from some users indicated that some positive reviews were given because they felt challenged when trying to perform the tasks (some evidently complicated), and expressions of joy were recorded when tasks were successfully completed.
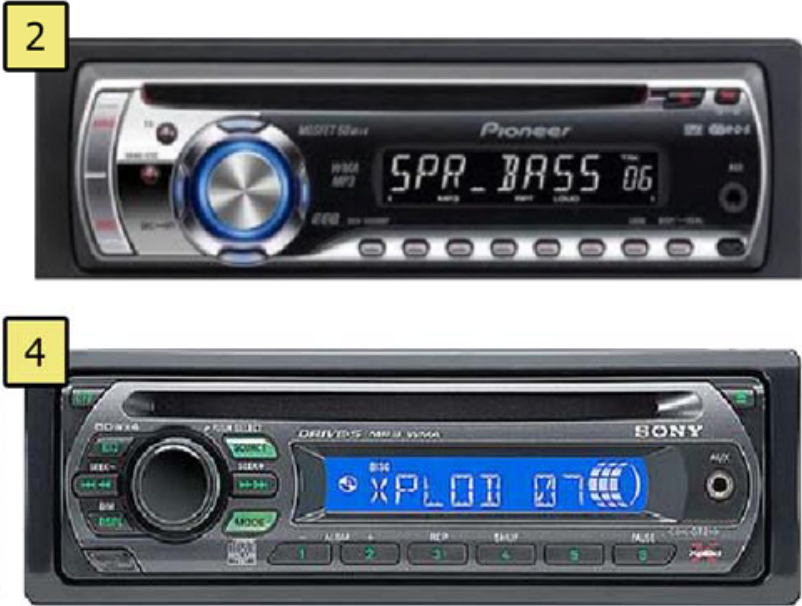

Fig. 1. Sound devices employed in the analysis: [1] Booster BDVX-5580MP; [2] Pioneer Mosfet50; [3] Pioneer DEH-2000MP; [4] Sony CTXGT210. 
Table 2 .

Scores for subjects' perception after use.

\begin{tabular}{lcccc}
\hline & A & B & C & D \\
\hline Satisfaction & 4,4 & 7,9 & 5,6 & 3,8 \\
\hline Ease of use & 2,6 & 6,2 & 5,4 & 2,5 \\
\hline Information comprehensibility & 3,0 & 4,3 & 4,1 & 3,5 \\
\hline Buttons layout & 4,1 & 5,7 & 3,4 & 3,0 \\
\hline Visual quality of the display & 3,1 & 7,3 & 3,9 & 3,1 \\
\hline Aesthetics & 6,1 & 7,9 & 2,8 & 2,2 \\
\hline A - Booster BDVX-5580MP
\end{tabular}

A - Booster BDVX-5580MP

B - Pioneer DEH-2000MP

C - Pioneer Mosfet50

D - Sony CTX-GT210

The product Sony CTX-GT210 showed the greatest usability problems in the sample. The great amount of errors and serious comprehensibility flaws led to a higher level of dissatisfaction among users.

\section{Final comments}

In general, the usability level of the products evaluated was low. The main problems identified in all models were difficult to fit the detachable front panel in the device; low readability of the information displayed in the models; functions that are not properly identified; there is an overloading of functions on the same buttons, which complicates or takes longer to operate; the information is commonly abbreviated or in codes and are presented only in English what was identified as incomprehensible for many users; there were found problems in the products layout, particularly regarding to the criteria of importance, sequence of operation and hierarchy of actions; some models also do not provide consistent feedback to users.

These problems would create a worse scenario if the users are also driving the vehicle (high demanding task). Since the problems identified were consistent for all products and most users, we can state that the redesign of automotive sound systems is essential to better fit to the basic usability requirements.

\section{References}

[1] Dumas, J. (2007) The great leap forward: The birth of the usability profession (1988-1993). Journal of Usability Studies, 2 (2), 54-60.

[2] ISO 9241-11 (1998) Ergonomic requirements for office work with visual display terminals (VDTs) - Part 11: Guidance on usability. International Standard Organization.
[3] Jordan, P. W. Human factors for pleasure in product use. Applied Ergonomics, vol. 29, n. 1, pp. 25-33, 1998.

[4] Nielsen, J. (1992). Finding usability problems through heuristic evaluation. Proceedings of the ACM Conference on Human Factors in Computing Systems, pp. 373-380. Monterey: ACM Press.

[5] Zwalen, H. T.; Adams, C. C.; Debald, D. P. Safety aspects of CRT Touch Panel Controls in Automobiles. In: Proceedings of the International Conference of vision in Vehicles, 2., Nottingham, 1987. Amsterdam: Elsevier, 1988, p. 1-10. 